\title{
Creating resilient SMEs: is business continuity management the answer?
}

\author{
R. Dahlberg ${ }^{1} \&$ F. Guay ${ }^{2}$ \\ ${ }^{1}$ Copenhagen Center for Disaster Research (COPE), Denmark \\ ${ }^{2}$ Danish Institute of Fire and Security Technology (DBI), Denmark
}

\begin{abstract}
According to the EU, during the past five years, small and medium enterprises (SMEs) have created $85 \%$ of new jobs and two-thirds of private sector employment in the region. SMEs are considered the backbone of the economy in Europe and represent more than $95 \%$ of enterprises in USA and Australia. They are considered more vulnerable to disasters because of their size. This paper argues, on the contrary, that SMEs also can be less vulnerable to sudden change than large corporations, drawing upon the ideas of Hayek and Taleb, and that networks of SMEs may contribute to the overall resilience of society if certain criteria are met. With this in mind, this paper will be examining how to create resilient SMEs. A well-known concept in the field is business continuity management. BCM is defined as "a holistic management process that identifies potential threats to an organization and the impacts to business operations those threats, if realized, might cause, and which provides a framework for building organizational resilience with the capability of an effective response that safeguards the interests of its key stakeholders, reputation, brand and valuecreating activities. Resilience, on the other hand, is a concept that has gained popularity in the past years and for which several definitions can be found. In 2009, the United Nations defines it as: "the ability of a system, community or society exposed to hazards to resist, absorb, accommodate to and recover from the effects of a hazard in a timely and efficient manner, including through the preservation and restoration of its essential basic structures and functions." This paper will define resilience and business continuity management by retracing the origins of both concepts through time. It will then compare them by highlighting their similarities and differences. Finally, it will provide recommendations on how SMEs can become more resilient.
\end{abstract}

Keywords: resilience, BCM, SMEs. 


\section{Introduction}

The European Union [1] has observed that during the past five years, small and medium enterprises (SMEs) have created $85 \%$ of new jobs and two-third of the total private sector employment in the EU. In the US, Grover and Suominen [2] report that SMEs make up 99 percent of all firms, employ over 50 percent of private sector employees, and generate 65 percent of net new private sector jobs. SMEs are important to our economies and these statistics demonstrate this fact well. Because they are so significant, they need to be promoted and protected. They can face many different challenges: economic crisis, new legislation, unstable market conditions, supply chain disruption, etc. In this paper, we will focus on disasters caused by natural hazards, which are also becoming a challenge for SMEs. An example is the Typhoon Haiyan which hit the Philippines in 2013 and destroyed many micro, small and medium sized enterprises. It was estimated that the overall damages to the economy were around 15 percent of the GDP in 2013. And for a region with 90 percent of SMEs and accounts 70 percent of global natural disasters [3], it becomes essential that their SMEs become more resilient. Ideas proposed by Hayek and Taleb can be applied to argue that network of SMEs could contribute to a more resilient society if certain criteria are met, which is an interesting theory that we will explore in this paper.

The purpose of this paper is to clarify what resilience signifies, in the context of small and medium enterprises, as well as how we can define business continuity management in that same context. By tracing the history of resilience and of business continuity management (BCM), we hope to clarify their significance for SME managers. We will then compare both concepts to highlight their differences and similarities, as well as exploring the hypothesis that SMEs can contribute to resilience. We will finally make some recommendations on how these concepts can be applied to the SMEs' contexts.

\section{Resilience}

\subsection{The evolution of resilience}

While the term has been used since Sir Francis Bacon's writings in the 17th century, "resiliency" and "resilience", deriving from the Latin resilire ("springing back"), has seen a dramatic increase in exposure over the last halfcentury, especially after 2005 [4]. Engineers of the 19th century constructed resilient structures such as bridges and buildings that were able to "bounce back" from sudden impact, and the concept has retained a meaning of "bounce back-ability", even if an important distinction between "ecological" and "engineered resilience" followed from C.S. Holling's writings on the subject beginning with a seminal paper in 1973 [5] and described in detail in 1996 [6]. Ecological resilience is an emergent property of complex ecosystems that do not evolve around a steady state like engineered resilient systems, but are governed by "dynamic equilibrium", meaning that impact or disturbance may flip the 
system into new stable domains. Therefore it doesn't make sense to talk about "bouncing back" in ecological resilience, as there is no normal to bounce back to, only "bounce forward" to a new configuration.

Since the UN Hyogo Framework for Action adopted the term resilience in its aims within global disaster risk reduction in 2005 [4], the concept has gained momentum in many different areas and disciplines such as safety engineering [7] sociology [8], governance [9], emergency preparedness [10] and urban development [11]. Psychologist have been using the term for decades to denote individuals as well as communities' ability to cope with shock and uncertainty [12], but especially in relation to communities the concept has grown in popularity lately.

The long history of the concept and ambitious projects such as the Rockefeller Foundation's "100 Resilient Cities", that aim at helping cities around the world become more resilient to the growing physical as well as social and economic challenges of the 21 st century, are signs that resilience is more than a buzzword. Still, resilience is a contested concept with many definitions and competing views from different disciplines. However, the "capacity of an individual, community or system to absorb and adapt in order to sustain an acceptable level of function, structure, and identity under stress" is a broad definition that captures the important aspects of resilience.

\subsection{Resilience for SMEs}

We have often been told that the size of SMEs make them more vulnerable because of their lack of resources. Research done by Alesch et al. [13] showed important findings. First, they could not find any statistical proof that traditional precautions such as structure protection, is a guarantee for business survival. Second, they found that most businesses do not fail right after a disaster, only the weak ones do. Third, most owners involved in their research had ideas on how to adapt with their new situation.

This tells us that SMEs are in a position to become more resilient, even though they might not have the resources large enterprises have. Research from Sullivan-Taylor and Branicki [14] studied the impact of sector, geographic location and industry upon organisational resilience and found that small firms can have an advantage over larger firms since they normally have less bureaucracy, the possibility of rapid decision making and rapid communications, as well as shorter processes. Stokes [15] also argues that smaller firms have to cope with more uncertainties and even though they can be perceived as more vulnerable and less tends to survive after a disaster, it might also be that they become more flexible and responsive. They become used to have to survive. So if we accept the fact that SMEs may already have some of the capabilities to cope with uncertain events, what should we be focusing on in order to increase their resilience?

Often "robustness" is confused with "resilience". Of course it is nice to be robust, but it is not the same as to be resilient. While robustness denotes a system or entity capable of withstanding shock so it isn't affected (the metaphor could be a tank with heavy armour deflecting a grenade), resilience is rather an 
emergent property of complex systems that enable them to absorb and adapt to sudden, unexpected change (here, the metaphor could be a network of soldiers each acting and reacting independently, but with a common goal, knowledge and language). In business terminology, a robust entity functions in a stable state with a well-defined "normal". When an unexpected perturbation occurs, such as natural hazard that influences the system's delivery of service, robustness will then mean that the system quickly bounces back to normal. Key elements in robustness are redundancy (stockpiles, multiple supply chains etc.). In resilience, however, the key elements are flexibility, adaptability and elasticity, allowing the system to retain its core functions and delivery of service by switching into a "new normal", i.e. a different stable state. Like a stream finds a new way to flow if you drop a big stone into it, a resilient system absorbs and adapts its way through times of crisis.

As stated above, resilience may be seen as an emergent property of a complex system, meaning that it is difficult to design a truly resilient system - it rather must evolve naturally from interactions between its constituent parts. But with regard to businesses it is possible to identify some aspects of systems that could be said not to contribute to the overall resiliency: Just in time-delivery, lean processes etc. that seek to reduce "slack" may add to the efficiency of business systems, but at the same time create vulnerabilities that will only reveal themselves in time of crisis, the point being that it is exactly "slack" that enables the system to absorb and adapt to unforeseen events.

This is not new thinking. The economist Friedrich A. Hayek created the Austrian school in Neoliberalist economic theory in the middle of the $20^{\text {th }}$ century, and a central element in Hayek's thinking was self-organizing economies: He saw markets, while exhibiting complex and thus unpredictable and uncontrollable behaviour, as able to self-regulate when exposed to sudden impact. Hayek rejected Keynesian economists' beliefs in an economic "stable equilibrium", not unlike Holling's distinction between engineered and ecological resilience [4].

Author of The Black Swan, Nassim Nicholas Taleb, uses a somewhat different vocabulary in the sequel to his bestseller, Antifragile: Things That Gain From Disorder, but with a similar meaning. Taleb talks, opposes "fragile" systems not with "robust", but with "anti-fragile" systems, a concept broadly synonymous with the modern understanding of resilience. An anti-fragile system not only absorbs stress, but also benefits from it - here we see the connection to adaptation in resilience. Taleb distinguishes between the engineered clockworklike mechanical systems that over time will be worn down and the more ecosystem-like system that constantly evolves (adapts) when faced with stress. He likens this process to the principle of "hormesis": that a small dose of a toxic agent may be good for an organism, i.e. because it triggers the immune system and thus protects the system against a larger dose at later stage. From this it follows that the overall anti-fragility/resilience of a system follows from a constant subjection to moderate amounts of stress. Too much will cause the system to collapse - but too little will create a standstill that over time results in weakness. 


\section{Business continuity management $(\mathrm{BCM})$}

With the evolution of crisis management activities, organisations need better plans and resources. "Organisations can face a wide variety of crises ranging from physical crisis such as accidents, product failure or loss of utilities (gas, power supply, water, telecommunications), personnel crises such as large scale staff illness or death, industrial action or staff criminality, external criminal crises such as terrorism and product tampering, information crises such a cybercrime or information theft, natural disasters such as flood and storms, economic crises such as economic recession, and reputational crisis such as internet defacement or malicious rumours" [16].

\subsection{The evolution of $\mathrm{BCM}$}

We can observe the evolution of business continuity management (BCM) by looking at all the legislation, national and international standards that have been introduced since the 1970s. According to Herbane [16], the first influence on the concept of BCM we know today, emerged from IBM computer model 360 and 370 , shading a light on the possible vulnerability of the use of data. He named this phase: emerging legislation phase (from mid-1970s to mid-1990s). Several Offices and Act were written in this period, where we can observe that business continuity was included in the legislation, like the US Expedited Funds Availability Act (1989) stating that is was a requirement for federal chartered financial institutions to have a business continuity plan in place.

The second period, emerging standard phase (mid-1990s to 2001), was characterized by standards coming from all sectors. For example, in 2000, The UK Ministry of Defence with the Joint service publication 503- business continuity management, or the NFPA 1600 Standards on disaster/emergency management and business continuity programs, just to name a few [16]. The standards introduced were more flexible and reviewable, and even local standards became international ISO Standards.

The third phase was launched by the terrorist attacks in September 2001, where it was now necessary to look into what we meant with BCM. Like Hill and Burgess highlight: "The attack on the World Trade Center on September 11, 2001 challenged the way many organizations thought about business continuity management $(\mathrm{BCM})$ and the assumptions on which their business continuity plans were based" [17]. We needed to include big human losses and psychological impacts in the vulnerability assessment. Several businesses also face the challenge that even thought they had BCP in place, activating these plans meant that they needed support from other services that could not keep up with the growing demand.

The post 9/11 period is regarded as a very fruitful period for the introduction of guidelines and regulations in the finance sector, with the public authorities, stock exchanges and utilities [16]. With the Business Continuity Institute publishing Good practice guidelines $(2002,2003)$ and 10 standards of professional competence (2003), it was becoming more clear that the role of 
individual specialist management skills would be pushing the standardisation at another level. Legislation and guidelines were starting to emerge from other countries such as Thailand, Singapore, Pakistan, Australia, and India.

This shift brings us to the last phase, the internationalisation phase. Standards and guidelines were created across disciplines and across countries: "the standards and guidelines that have emerged in the internationalisation phase recognise the importance of collaboration between organisations in crisis response and harmonise the quality and nature of practices within them" [16].

National standards were becoming international standards and new standards were emerging from international bodies such as ISO. The best example is the ISO standard 22313:2012 Societal security - Business continuity management systems [18]. With this standard, came the PDCA model (Plan, Do, Check and Act) which include policies, people, processes and documentation present within an organization. ISO chose this model in order to assure consistency with other management system standards, and so that it can be applied to any organisation wishing to implement $\mathrm{BCM}$.

Now that we have been through the history of business continuity management, we would like to investigate how BCM is being applied within SMEs.

\subsection{BCM for SMEs}

Research shows that there is little done on SMEs and concepts like crisis management, business continuity management or disaster recovery Herbane [19]. Hill and Burgess [17] found that there were two approaches to BCM: one is the crisis management approach; the second is the risk management approach. The crisis management approach of BCM takes into account all organizational processes and the individuality of each organization. It considers that business interruptions have social and technical characteristics, that organizations can be responsible of their own failure, that resilience can be built through processes and procedures, and that a disaster can have an impact on stakeholders inside and outside the organization. The risk management approach is based on the "five A's" of risk management: assessing risk; accepting or rejecting risk; avoid, transfer or reduce risk; analyse performance gaps; act to improve.

The two approaches are quite different, but they were summarize by Hill and Burgess [17] by saying that the crisis management approach is more proactive, because it recognizes the fact that disaster's effects can be reduced if organization can identify the signs. And the risk management approach is more reactive because it is based on the organisation's previous risk assessments, previous interruptions and previous actions. Either approaches can be applied to SMEs, but when choosing BCM, one must also be aware of some general challenges. For example, the fact that very detailed plan can sometimes be less effective, or that the expectation of what was possible after a disaster were somehow unrealistic. Hill and Burgess [17] also raise three other issues: information technology, personnel and management. Business Continuity plans do not necessarily consider the fact that several building or several services can be affected at the same time; or that personnel can be missing or under shock. 
Organisations also need the support of the senior management in order to implement BCM, and it is even suggested to involve employees in the process to insure that they understand the concept and are better prepared for an event.

BCM can be a good solution for creating more resilient SMEs, but it is important to be aware of the weaknesses of this concept and assure that other measures are taken.

\section{Linking resilience and $\mathrm{BCM}$}

1. In the field of resilience, crisis management and BCM, one other concept which has been used when considering SMEs is: organisational resilience. In Sullivan-Taylor and Branicki's perspective, organisational resilience investigates why some types of organisation have very low rate of failure [14]. Examining fours factors that contribute to organisational resilience, their findings were that:

2. Resourcefulness was an important barrier to SMEs' resilience, related to identifying problems, prioritizing and mobilising resources;

3. Technical systems were not a major priority for SMEs;

4. SME managers prefer talking about "muddling through" and question taking action;

5. SMEs have a positive potential for quick response and flexibility.

6. The results of this research demonstrate that SMEs have challenges as well as advantages when it comes to resilience. We believe that with the increasing complexity and frequency of unexpected events, we will need a better answer than "muddling through". Business continuity management, as mentioned earlier, is not the perfect solution, but it does provide a more structured and proactive approach.

We also need to consider the difference between the SMEs, by their nature but also capacities. "In the context of resilience on size of regulation or advice arguably does not fit all and it is argued that this is due to the impact of organisational size upon the type of resilience capabilities that an organisation might possess" [14].

Resilience is, as mentioned above, in its ecological sense an emergent feature of complex systems. With this in mind, it is necessary not to look at SMEs as single, independent business entities, but rather as interdependent business network nodes that interact. The resilience of the complex structure as a whole emerges from the ability of individual nodes to "fail successfully", i.e. without causing the entire system to collapse.

But a network of SMEs does not necessarily become resilient on its own. Judith Rodin from the Rockefeller Foundation lists 5 generic measures of resilience: 1) Awareness, 2) Diversity, 3) Integration, 4) Self-regulation, and 5) Adaptation. 1) For a business network of SMEs to be aware requires constant monitoring in order to maintain an understanding of the system's strengths and weaknesses. 2) Diversity means that SMEs should connect with entities from other business areas, geographical locations and cultural traditions to ensure access to multiple solutions to problems. 3) An integrated network of SMEs is 
able to efficiently share information and coordinate joint action. 4) Selfregulation means that the network can avoid cascading effects by containing failure. 5) The "adaptiveness" of the system denotes its ability to solve problems by developing new plans or modifying behaviour under pressure [11].

In an uncertain world, inhabited by Black Swans and ravaged by Perfect Storms, nothing is too big to fail. This was proved by the failing of Lehman Brothers (2008), which at more than $\$ 600$ billion in assets stands as the largest bankruptcy in U.S. business history. But perhaps SMEs can be small enough to succeed if they engage in networks that enhance their joint awareness, diversity, integration, self-regulation and adaptive capabilities.

\section{Recommendations}

So what are our recommendations in order to create more resilient SMEs? Considering that $\mathrm{BCM}$ is part of the solution, we would have to mention that first, SMEs should be proactive. In order to be able to react better to disasters, SMEs need to recognize that the risk is present and do something about it. Burke et al. [20] even mention that not only being proactive but promoting resilience is the key.

Second, SMEs have to be realistic as to what they can plan for and what will be possible post-disaster. As Tierney states [8], "disasters do no follow preordained scripts. Even in situations where there is extensive disaster experience, those seeking to respond invariably confront unforeseen situations." When SMEs do plan, they should be aware that unexpected events means that their plan might not be realizable and evaluate what they consider as crucial for the survival of the enterprise after a disaster.

Third, SMEs should do a risk assessment to assess the potential impacts of various events on all levels; for example, assessing the vulnerabilities of an infrastructure towards different types of events, different locations or different activities, as also mentioned under the "diversity" and "adaptiveness" measures earlier.

Fourth, SMEs should not underestimate the importance of the people factor. As Hill and Burgess [17] state in their paper, one of the most important lessons of September 11 is that plans are often made on the assumption that all employees will be available after a disaster. The reality is that it is not always the case and that we can't predict how employees will react in stressful situations.

\section{Conclusion}

This paper defined resilience and business continuity management by considering the origins of both concepts. Investigating the provenance of these concepts facilitated the understanding of today's definitions. This paper also identified how resilience and BCM can be applied to SMEs. Linking both concepts showed that they are not incompatible, but that BCM is not the only tool to apply in order to create for resilient SMEs. What is certain is that finding 
one unique solution for all SMEs is not possible, as it will depend on each individual enterprise, and their level of awareness, diversity, self-regulation, integration and adaptive capabilities. We concluded with a few recommendations on what SME managers should consider together with a BCM approach, if they want to be successful at becoming more resilient.

\section{References}

[1] EU, http://ec.europa.eu/growth/smes/index_en.htm

[2] Grover, A. \& Suominen, K., 2014 Summary - State of SME Finance in the United States, Trade Up, 2014.

[3] APEC, http://www.apec.org/Press/Features/2014/0324_smes.aspx

[4] Dahlberg, R., Tind Johannessen-Henry, C., Raju, E. \& Tulsiani, S., Resilience in disaster research: three versions, Civil Engineering and Environmental Systems, 32:1-2, pp. 44-54, 2015.

[5] Holling, C.S., Resilience and stability of ecological systems, Annual Reviews of Ecological Systems, 4:1-23, 1973.

[6] Holling, C.S., Engineering Resilience versus Ecological Resilience, Engineering Within Ecological Constraints, National Academy Of Engineering, National Academy Press; Washington DC, 1996.

[7] Hollnagel, E., Woods, D.D., \& Leveson, N., Resilience Engineering. Concepts and Precepts, Ashgate: Farnham, 2006.

[8] Tierney, K., The Social Root of Risk: Producing Disasters, Promoting Resilience. Stanford University Press: California, 2014.

[9] Chandler, D., Resilience, The governance of complexity, Routledge, 2014.

[10] National Research Council: Disaster Resilience: A National Imperative, The National Academies Press: Washington DC, 2012.

[11] Rodin, J., The Resilience Dividend: Managing disruption, avoiding disaster, and growing stronger in an unpredictable world. Profile Books: London, 2015.

[12] Kolar, K., Resilience: Revisiting the Concept and its Utility for Social Research, International Journal of Mental Health and Addiction, 9, pp. 421-433, 2011.

[13] Alesch, D.J., Holly, J.N., Mittler, E. and Nagy R. Organizations at Risk: What Happens When Small Businesses and Not-for-Profits Encounter Natural Disasters, Public Entity Risk Institute: VA, 2001.

[14] Sullivan-Taylor, B. and Branicki, L., Creating resilient SMEs: why one size might not fit all, International Journal of Production Research, 49(18), pp. 5565-5579, 2011.

[15] Stokes, D., Understanding the Small Business. London: Thomson, 2002.

[16] Herbane, B., The evolution of business continuity management: A historical review of practices and drivers, Business History, 52(6), pp. 9781002, 2010.

[17] Hill, R. \& Burgess, S., Issues in Business Continuity Management, Idea group publishing: USA, pp. 287-289, 2003. 
984 Sustainable Development, Vol. 2

[18] ISO (2012) ISO 22301:2012 Societal security - Business continuity management system. Online. https://www.iso.org/obp/ui/\#iso:std:iso: 22301:ed-1:v2:en.

[19] Herbane, B., Small Business Research: Time for a Crisis-Based View, International Small Business Journal, 28(1), pp. 43-64, 2010.

[20] Burke, S., Wilson, K., and Salas, E., The use of a team-based strategy for organizational transformation: guidance for moving toward a high reliability organization, Theoretical Issues in Ergonomics Science, 6(6), pp. 509-530, 2005. 